\title{
Design Space para Jornalismo de Dados Data Journalism Design Space
}

\author{
Tajla Medeiros \& Virgínia Tiradentes Souto
}

jornalismo de dados, design space, design de informação

\begin{abstract}
O Jornalismo de Dados pode ser entendido como uma forma de contar histórias em que os métodos tradicionais do jornalismo são combinados a análise de dados, programação e técnicas de visualização (Appelgren e Nygren, 2014). Apesar do acentuado crescimento da popularidade do Jornalismo de Dados nos últimos anos, as publicações acadêmicas sobre contar histórias de dados (em inglês, data storytelling) ainda são incipientes (Segel e Heer, 2010; Hewett, 2016; Ojo e Heravi, 2018). Neste contexto, o presente artigo busca contribuir com esta área do conhecimento por meio da revisão dos estudos que tratam da ferramenta conceitual design space aplicada às histórias de dados, explorando as opções de design que permeiam a elaboração desses conteúdos.
\end{abstract}

data journalism, design space, information design

Data Journalism can be understood as a way of telling stories in which traditional methods of journalism are combined with data analysis, programming and visualization techniques (Appelgren and Nygren, 2014). Despite the strong growth in the popularity of Data Journalism in recent years, academic data storytelling publications are still incipient (Segel and Heer, 2010, Hewett, 2016, Ojo and Heravi, 2018). In this context, the present article seeks to contribute to this area of knowledge by reviewing the studies that deal with the conceptual design space tool applied to the data stories, exploring the design options that permeate the elaboration of these contents.

\section{Introdução}

O Jornalismo de Dados pode ser entendido como uma forma de contar histórias em que os métodos tradicionais do jornalismo são combinados a análise de dados, programação e técnicas de visualização (Appelgren \& Nygren, 2014). Apesar do acentuado crescimento da popularidade do Jornalismo de Dados nos últimos anos, as publicações acadêmicas sobre contar histórias de dados (em inglês, data storytelling) ainda são incipientes (Segel \& Heer, 2010; Hewett, 2016; Ojo \& Heravi, 2018).

Se, de um lado, entende-se que 'o Jornalismo de Dados é uma vertente da Reportagem Assistida por Computador porque envolveria não apenas o uso de computadores, mas o conhecimento mínimo em estatística, sistemas computacionais e métodos das ciências sociais' (Mancini \& Vasconcellos, 2016, p. 73); de outro, podemos afirmar que o storytelling, característica essencial desse gênero, o distancia de seu antecessor, aproximando-o de narrativas literárias. Historicamente, as análises sobre as confluências entre jornalismo e narrativas ou storytelling sempre tiveram divergências, dificultando a formação de referenciais teóricos para o tema (Groot Kormelink \& Costera Meijer, 2015).

Neste contexto, o presente artigo busca contribuir com esta área do conhecimento por meio da revisão dos estudos que tratam da ferramenta conceitual design space aplicada às histórias de dados, explorando as opções de design que permeiam a elaboração desses conteúdos.

\section{Design Space para Jornalismo de Dados}

Jornalismo de Dados pode ser entendido como uma forma de contar histórias em que os

Anais do 9 CIDI e 9 CONGIC

Luciane Maria Fadel, Carla Spinillo, Anderson Horta,

Cristina Portugal (orgs.)

Sociedade Brasileira de Design da Informação - SBDI

Belo Horizonte | Brasil | 2019

ISBN $978-85-212-1728-2$
Proceedings of the 9th CIDI and 9th CONGIC

Luciane Maria Fadel, Carla Spinillo, Anderson Horta,

Cristina Portugal (orgs.)

Sociedade Brasileira de Design da Informação - SBDI

Belo Horizonte | Brazil | 2019

ISBN 978-85-212-1728-2 
métodos tradicionais do jornalismo são combinados a análise de dados, programação e técnicas de visualização. Essas histórias são normalmente produzidas a partir do uso de grandes quantidades de dados públicos ou coletados com o auxílio das pessoas em geral prática conhecida como crowdsourcing (Appelgren \& Nygren, 2014). O livro The Data Journalism Handbook (Gray, Chambers \& Bounegru, 2012) traz importante sistematização para a área, contendo a visão de especialistas e a análise de exemplos praticados em diversos países. Na seção dedicada a explicar o termo, Paul Bradshaw não elabora conceituação sintética e objetiva, mas apresenta as principais características que diferenciariam esse gênero do jornalismo dos demais. Para o autor, dados e tecnologia seriam elementos-chave, devendose entender, primeiramente, que, hoje, quase tudo poderia ser entendido como dado, uma vez que todas as informações digitais, a despeito de seu formato e conteúdo, podem ser descritas por números binários (zero e um). Essa característica digital das informações, aliada a sofisticadas e acessíveis ferramentas de manipulação e edição de dados, dariam enormes possibilidades à prática jornalística, em suas diferentes fases: a) pesquisa (e.g., automatização da apuração); b) elaboração (e.g., processamento e interpretação de milhares de centenas de dados); e c) apresentação (e.g., elaboração de infografias interativas).

Entende-se que o predecessor do Jornalismo de Dados teria sido a Reportagem com Auxílio do Computador - RAC, do inglês Computer Assisted Reporting - CAR, conceito cunhado pelo jornalista americano Philip Meyer nos anos 1960s (Gray, Chambers \& Bounegru, 2012; Howard, 2014; Coddington, 2015; Knight, 2015). Pouco tempo depois, Meyer teria apresentado o termo Jornalismo de Precisão (em inglês: Precision Journalism) e publicado instruções para que jornalistas aplicassem métodos científicos na coleta e análise de dados para apresentação de notícias. A visão era de que o rigor científico aplicado à prática profissional levaria a reportagens mais assertivas e acuradas (Meyer, 1973). No fim do século XX e início do XXI, o tema volta a ter relevância: com o aumento do uso de microcomputadores nas redações, surgem novas pesquisas sobre computação e práticas jornalísticas, tratando da inclusão de conhecimentos sobre ciências, dados e tecnologia no currículo de jornalistas (Williams, 1997; Davenport, Fico e DeFleur, 2002); entre os pesquisadores, a preferência era pela terminologia Reportagem com Auxílio do Computador, em detrimento de Jornalismo de Precisão (Träsel, 2014). Nos últimos anos, novamente o tema recebe atenção de pesquisadores do jornalismo, que agora adotam terminologias diversas para práticas muitas vezes 'ambiguamente relacionadas', conforme aponta Coddington (2015). São exemplos: Jornalismo Computacional, Jornalismo de Dados, Jornalismo Guiado por Dados e Jornalismo Digital em Base de Dados. A esse grande campo de estudos sobre o uso de algoritmos, dados e métodos das ciências sociais para fins de reportagens, narrativas e histórias jornalísticas, Gynnild (2014) propõe a terminologia Exploração Computacional no Jornalismo - ECJ (tradução nossa, do inglês Computational Exploration in Journalism - CEJ), significando 'o processamento inovador que ocorre na interseção entre jornalismo e tecnologia de dados' (tradução nossa, Gynnild, 2014, p. 715).

Exemplificamos como as diferentes terminologias para os usos computacionais no jornalismo empregadas na literatura. Träsel utiliza Jornalismo de Dados e Jornalismo Guiado por Dados - JGD como sinônimos, significando 'produção, tratamento e cruzamento de grandes quantidades de dados, de modo a permitir maior eficiência na recuperação de informações, na apuração de reportagens a partir de conjuntos de dados, na circulação em diferentes plataformas (computadores pessoais, smartphones, tablets) e na geração de visualizações e infografias' (Träsel, 2014, p. 296). Já Mancini e Vasconcellos (2016) propõem a diferenciação entre o que é Jornalismo de Dados e o que se trataria apenas de Jornalismo com Dados, de acordo com o nível em que as seguintes dimensões estariam presentes na reportagem: investigativa (busca por dados e revelações), interpretativa (exposição das relações entre os dados) e comunicativa (visualizações das informações). Quanto melhor explorasse as dimensões, mais um conteúdo se caracterizaria como Jornalismo de Dados. Berret, Phillips e Coll (2016) entendem que tanto o Jornalismo de Dados quanto o Computacional podem ser entendidos como 'uso de dados para fins jornalísticos de pesquisa e apresentação de histórias de interesse do público' (tradução nossa, 2016, p. 9), mas que o último seria diferenciado pelo maior uso de algoritmos e aprendizado de máquina. Ojo e Heravi (2018) assumem como sinônimo os termos Reportagem com Auxílio do Computador, Jornalismo Computacional e Jornalismo de Dados. Por outro lado, alguns autores entendem que o Jornalismo Computacional, diferencia-se da RAC e do Jornalismo de Dados, pela predominância não só 
da aplicação de computação nas práticas jornalísticas, mas também - e, talvez, principalmente - do pensamento computacional (Diakopoulos, Nicholas, 2011; Stavelin, 2014; Coddington, 2015), entendido como a resolução de problemas, o desenho de sistemas e a compreensão do comportamento humano a partir de conceitos e ferramentas da Ciência da Computação (Wing, 2006). Ainda que esteja entre esse último grupo de pesquisadores, Coddington (2015) entende que, quando analisado sob o ponto de vista das práticas e relações profissionais, da cultura de dados abertos e da relação com o público, a Reportagem com Auxílio de Computador se distancia e o Jornalismo de Dados e o Jornalismo Computacional se aproximam. Já Stavelin (2014) entende que todas as denominações se referem a práticas jornalísticas que utilizam softwares como ponto de partida para acessar e manipular dados, mas cada uma possui sua especificidade: o Jornalismo de Precisão enfatizaria o uso do método científico; a RAC, o uso de ferramentas digitais; o JDBD, a estruturação do armazenamento e da recuperação da informação (por isso, também chamado de Jornalismo Estruturado); o JD, a análise e apresentação de dados, com grande foco em construção de narrativas; o JGD, a busca de revelações a partir da análise de bancos de dados; e o Jornalismo Computacional, a combinação de valores e fatores das áreas de jornalismo e computação para criação de ferramentas e aplicação de métodos - o único a poder elaborar softwares como produto final.

Para os fins desse artigo, importa apenas o resultado final dessas práticas jornalísticas. Assim, utilizaremos os termos Jornalismo de Dados, Jornalismo Guiado por Dados e Jornalismo Computacional indistintamente, conforme entendimento de que, em geral, os objetivos são os mesmos (Ojo \& Heravi, 2018; Young, Hermida \& Fulda, 2018): contar histórias por meio dos métodos tradicionais do jornalismo, combinados a análise de dados, programação e técnicas de visualização (Appelgren \& Nygren, 2014). Daremos, todavia, preferência a Jornalismo de Dados, pela maior disseminação do termo entre os profissionais e pesquisadores de design space no tema. Endossamos o entendimento de Stavelin (2014), para quem a referida terminologia é a que melhor representa as pesquisas que focam em apresentação de dados e construção de narrativas, aspectos essenciais do design space construído neste trabalho.

Para além da terminologia, importa para esta pesquisa um aspecto central desse tipo de reportagem: a prática de contar histórias por meio de abordagem estruturada e utilizando ferramentas visuais e recursos narrativos para comunicar ideias a partir de dados (Dykes, 2016; Ojo \& Heravi, 2018). Se, de um lado, entende-se que 'o Jornalismo de Dados é uma vertente da Reportagem Assistida por Computador porque envolveria não apenas o uso de computadores, mas o conhecimento mínimo em estatística, sistemas computacionais e métodos das ciências sociais' (Mancini \& Vasconcellos, 2016, p. 73); de outro, podemos afirmar que o storytelling o distancia de seu antecessor, aproximando-o do Jornalismo Longform Multimídia. Para a professora Meredith Broussard ${ }^{1}$, para se tornar um bom jornalista de dados é preciso aprimorar as técnicas de storytelling, experimentar diferentes formas de contar histórias para o público, e entender os dados - que, enfatiza a professora, 'também seriam criados por pessoas'. Já Howard (2014) utiliza as expressões 'narrativas poderosas' e 'técnicas criativas de storytelling' ao lado de 'pensamento rigoroso e planejado' para descrever processos e práticas do jornalismo contemporâneo (tradução nossa, p. 18).

Apesar do acentuado crescimento da popularidade do Jornalismo de Dados nos últimos anos, as publicações acadêmicas sobre contar histórias de dados (em inglês, data storytelling) ainda são incipientes (Segel \& Heer, 2010; Hewett, 2016; Ojo \& Heravi, 2018). Historicamente, as análises sobre as confluências entre jornalismo e narrativas ou storytelling sempre tiveram divergências, dificultando a formação de referenciais teóricos para o tema (Groot Kormelink \& Costera Meijer, 2015). A despeito disso, a aplicação de estilos narrativos em diversos formatos noticiosos são tendência nas práticas do jornalismo contemporâneo (Shim, 2014; Groot Kormelink \& Costera Meijer, 2015) e, entre idas e vindas, são utilizados nos formatos noticiosos desde o século XVIII, com o surgimento do Jornalismo Literário (Kerrane \& Yagoda, 1998; Jacobson, Marino \& Gutsche, 2016). Groot Kormelink \& Costera Meijer (2015) entendem que as discussões acadêmicas na área fizeram pouco progresso ao longo das últimas décadas

\footnotetext{
${ }^{1}$ Em: Howard, 2014.
} 
devido a oposições - que os autores entendem como refutáveis - de espaços discursivos. De um lado, a noção de que notícias e reportagens devem ser objetivas e tratar apenas de fatos, sem o menor sinal de aspectos relacionados a experiências, emoções e vida privada e cotidiana; de outro, radical negação à percepção, considerada ultrapassada, de que jornalismo seria primariamente caracterizado por objetividade, imparcialidade e representação acurada da realidade. Sobre o assunto, estudos como os dos autores Nossek \& Berkowitz (2006) apontam como a influência da cultura e da subjetividade confere mesmo às consideradas 'notícias sérias' (em inglês: hardnews) aspectos simbólicos típicos de narrativas de ficção.

Nesse contexto, a análise do design space adquire grande relevância para um produto de Comunicação que tende a misturar a objetividade e capacidade analítica das Ciências de Dados com a criatividade e inventidade de narrativas fílmicas e literárias, assunto que abordamos no tópico a seguir. Por favor, siga as instruções abaixo para formatar o layout de seu artigo. Este documento mostra como deve ser a formatação do artigo.

\section{Design space}

Design space pode ser entendido como ferramenta conceitual que explora propostas possíveis de design, sendo usado tanto para a prática profissional do design quanto para entender seus processos, podendo ser espaço extremamente complexo e multidimensional com número infindável de soluções (Westerlund, 2009). Estudos e análises sobre o design space têm como objetivo conectar as preocupações relativamente teóricas aos aspectos práticos do design. Aplicada à área de pesquisa aqui estudada, a análise do design space é importante para que os resultados dos estudos sobre elaboração de data storytelling sejam incorporados às práticas profissionais do Jornalismo de Dados, área caracterizada pela rápida e constante modificação. A abordagem ainda é incipiente na literatura de histórias de dados (Conlen \& Heer, 2018; Ojo \& Heravi, 2018) e é possível verificar uma lacuna entre as diretrizes acadêmicas de visualização e sua aplicação no mundo real (Moritz et al., 2018).

Embora não haja apenas uma instrução sobre como o design space deva ser elaborado, podendo variar de produto a produto, destacamos a proposta de MacLean et al. (1991), que pode ser resumida pela notação QOC - Questões, Opções e Critérios. Questões representariam os elementos chaves de design; Opções proveriam as possibilidades para responder a essas questões; já Critérios permitiriam entender e comparar as diferentes opções da mesma questão.

$\mathrm{Na}$ figura 1, vemos uma representação de design space QOC. As questões são iniciadas por 'Q:'; as opções, por 'O:'; e, os critérios, por 'C:'. Os traços contínuos que ligam opções a critérios indicam correspondência entre as ideias; os descontínuos, o contrário. Para evitar violação das leis de direitos autorais, por favor, não utilize longas e muitas citações de uma mesma fonte, ou figuras publicadas previamente sem um documento de autorização de uso dos direitos autorais. Isto também se refere a imagens produzidas por você autor, mas que já tenham sido publicadas em outro veículo e que o seu direito autoral tenha sido transferido à editora. Autores que não fornecerem documentos de autorização de uso de direitos autorais terão seus artigos devolvidos. Nós trataremos rigorosamente violações de direitos autorais..

Utilizando a notação criada por MacLean et al. (1991), podemos dizer que os design spaces que encontramos na literatura sobre histórias de dados trabalham apenas com questões - sendo comumente chamadas de dimensões - e opções, mas não exploram sistematicamente os critérios, o que não permite explorar a justificativa de design (ou design rationale) para os conteúdos elaborados. 
Figura 1: Representação de fragmento de design space usando o método QOC, onde analisam-se possibilidades para barra de rolagem de um website (adaptado, pela autora, de MacLean et al., 1991).

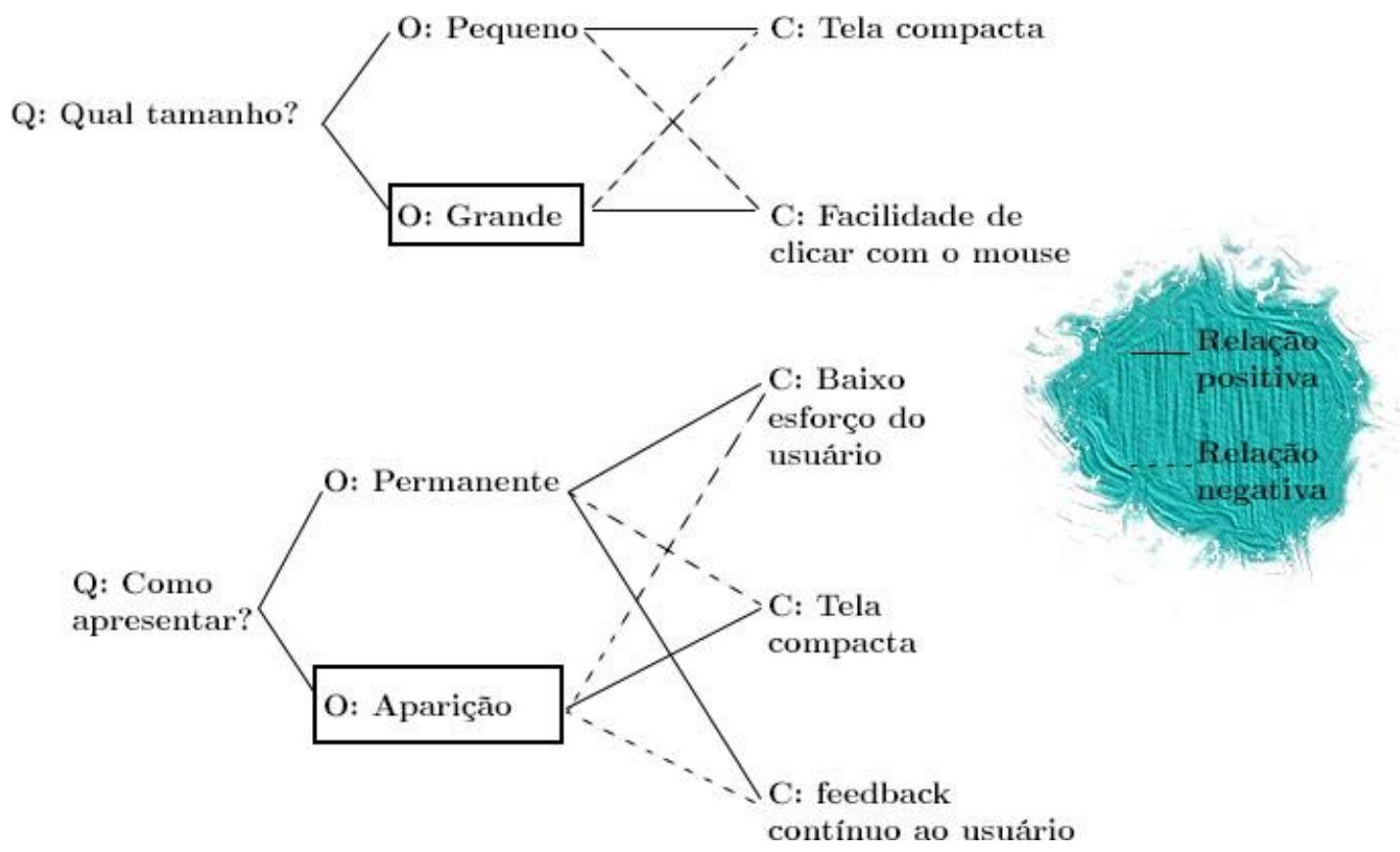

Um dos primeiros estudos que aborda o design space no Jornalismo de Dados foi o de Segel e Heer (2010). Buscando 'identificar e categorizar as características que efetivamente permitem contar histórias com dados' (tradução nossa, p. 1140), o estudo teve como resultado relevante a sistematização das técnicas narrativas utilizadas por uma amostra de 80 conteúdos. Entendendo que não era possível caracterizar completamente os diferentes fluxos de narrativas visuais com o modelo de Segel e Heer, os autores McKenna et al. (2017) revisam a proposta para incluir o que os autores denominam de flow factors, que são informações sobre como o usuário interage com histórias de dados visuais: entrada de navegação, nível de controle, progresso da navegação, layout da história, papel da visualização, progressão da história e feedback de navegação, conforme vemos na tabela 1, abaixo.

Tabela 1: Design space do fluxo narrativo de histórias de visualização de dados (adaptado, pela autora, de McKenna et al., 2017).

\begin{tabular}{l|l|l|l} 
Dimensão & Descrição & Opções & Valor \\
\hline Input de navegação & $\begin{array}{l}\text { Analisa como um leitor } \\
\text { interage para progredir } \\
\text { em uma narrativa de } \\
\text { visualização }\end{array}$ & $\begin{array}{l}\text { Rolagem, botão ou } \\
\text { slider }\end{array}$ & Presente ou ausente \\
\hline Nível de controle & $\begin{array}{l}\text { Analisa quanto controle } \\
\text { um leitor tem sobre o } \\
\text { movimento ou as } \\
\text { transições animadas } \\
\text { que compõem a } \\
\text { história }\end{array}$ & $\begin{array}{l}\text { Texto, visual e } \\
\text { animações }\end{array}$ & $\begin{array}{l}\text { Contínuo, discreto ou } \\
\text { híbrido }\end{array}$ \\
\hline Progressão da & $\begin{array}{l}\text { Analisa como o leitor } \\
\text { percebe o estágio em } \\
\text { que está na história }\end{array}$ & $\begin{array}{l}\text { Texto, pontos, visual e } \\
\text { outros }\end{array}$ & $\begin{array}{l}\text { Presente, parcialmente } \\
\text { presente ou ausente }\end{array}$ \\
\hline Layout da história & $\begin{array}{l}\text { Corresponde tanto ao } \\
\text { tipo de modelo de } \\
\text { layout quanto ao nú- } \\
\text { mero de colunas } \\
\text { usadas na história }\end{array}$ & $\begin{array}{l}\text { Documento, slides ou } \\
\text { colunas }\end{array}$ & $\begin{array}{l}\text { Exclusivo, parcial ou } \\
\text { ausente }\end{array}$ \\
\hline
\end{tabular}

Anais do 9 Congresso Internacional de Design da Informação | CIDI 2019 


\begin{tabular}{l|l|l|l} 
Papel da visualização & $\begin{array}{l}\text { Analisa o nível de } \\
\text { importância e o } \\
\text { propósito da } \\
\text { visualização na história }\end{array}$ & $\begin{array}{l}\text { Igual, figura ou } \\
\text { anotação }\end{array}$ & Sim ou não \\
\hline Progressão da história & $\begin{array}{l}\text { Categoriza os } \\
\text { caminhos possíveis da } \\
\text { história }\end{array}$ & Linear, skip ou outro & Apenas uma opção \\
\hline $\begin{array}{l}\text { Feedback de } \\
\text { navegação }\end{array}$ & $\begin{array}{l}\text { Analisa como é } \\
\text { mostrado aos leitores } \\
\text { que o input afeta a } \\
\text { história. Combina } \\
\text { transições animadas } \\
\text { com animações do } \\
\text { texto da história ou } \\
\text { outros componentes, } \\
\text { como o fading ou } \\
\text { movimento }\end{array}$ & $\begin{array}{l}\text { 1)Textual, visual ou } \\
\text { widget }\end{array}$ & $\begin{array}{l}\text { 2) Ordem } \\
\text { parcialmente presente } \\
\text { ou ausente }\end{array}$ \\
\hline
\end{tabular}

Além dos fluxos narrativos, também recebem destaque dos estudos de design space as tecnologias que são utilizadas (Segel \& Heer, 2010; Parasie \& Dagiral, 2013; Bakker, 2014; Gynnild, 2014; Fink \& Anderson, 2015; Uskali \& Kuutti, 2015; Berret, Phillips \& Coll, 2016; Müller et al., 2016; Hewett, 2016). Sistemas, ferramentas e linguagens de computação aplicadas à elaboração de histórias de visualizações de dados são componentes fundamentais desse tipo de design space, e 'um desafio para os pesquisadores desse espaço é que as ferramentas e as técnicas utilizadas pelos praticantes estão constantemente mudando' (tradução nossa, Conlen \& Heer, 2018, p. 2). O uso de ferramentas e tecnologias computacionais no Jornalismo de Dados é tão intenso, que Fink e Anderson (2015) falam em jornalistas-programadores para definir esses novos profissionais.

Para facilitar o acesso de produtores de conteúdos a essas ferramentas, estudos e ferramentas têm sido desenvolvidos. O estudo e desenvolvimento do Draco, software de acesso livre para elaboração automatizada de visualizações de dados, por exemplo, busca transformar o conhecimento teórico do design em ferramenta concreta e testável (Moritz et al., 2018); e da proposição da ferramenta Idyll, que busca reduzir o esforço em codificação customizada para elaborar artigos interativos e servir como plataforma para que 'pesquisadores coletem e analisem dados sobre como usuários interagem com as histórias' (Conlen \& Heer, 2018).

Neste tópico, buscamos enfatizar os vários papéis do design space das histórias de dados, que compreendem o mapeamento das técnicas e ferramentas mais utilizadas, facilitando o acesso do profissional a esse conhecimento. A seguir, propomos reflexões sobre a literatura e apontamos caminhos futuros de pesquisa.

\section{Considerações finais}

Novas técnicas e tecnologias abriram possibilidades para novos caminhos no jornalismo, permitindo o surgimento do Jornalismo de Dados, que permite contar histórias por meio dos métodos tradicionais do jornalismo, combinados a análise de dados, programação e técnicas de visualização (Appelgren \& Nygren, 2014). Para compreender as técnicas e as ferramentas aplicadas, pesquisadores têm se dedicado a compreender o design space desses conteúdos.

Uma primeira dificuldade na literatura é a própria conceituação de história de dados. Uma crítica à questão foi feita por Lee et al. (2015), que entendem que ao utilizar definição mais ampla, 'qualquer imagem contendo gráficos simples com algumas explicações pode ser chamada história visual de dados' (tradução nossa, 2015, p.85). Buscando estabelecer uma referência para o conceito, os autores propõem que seja categorizado desta forma apenas o objeto que apresentar: fatos específicos apoiados por dados; anotações ou narrações que claramente enfatizem as mensagens e intenções da comunicação; e organização das informações para suportar objetivos de comunicação bem elaborados. Entendemos que a

Anais do 9 Congresso Internacional de Design da Informação | CIDI 2019

Proceedings of the 9th Information Design International Conference

Anais do $9^{\circ}$ Congresso Nacional de Iniciação Científica em Design da Informação | CONGIC 2019

Proceedings of the $9^{\text {th }}$ Information Design Student Conference 
tentativa de conceituação ainda é vaga e chama atenção para necessidade de amadurecimento do tema.

Outro ponto de atenção seria a utilização de design spaces sem critérios, ou seja, sem analisar a justificativa de design que está por trás das escolhas feitas. A primeira e mais óbvia razão para a ausência de 'critérios' é que a análise dos design spaces que encontramos na literatura não são feitas pelos profissionais que elaboraram as narrativas. Para além disso, entendemos que o design space dos conteúdos de Jornalismo de Dados possuem peculiaridades similares às que Brath e Banissi (2016) identificaram nas ferramentas conceituais dos sistemas de visualização de informação. Os autores explicam que, em geral, uma ferramenta conceitual é importante para fornecer a consistência que torna o design de artefatos em geral mais eficientes e previsíveis. Exemplo: ao construir uma fonte tipográfica há certo consenso de que as letras devem ter a largura, os terminais e a altura-x similares, para facilitar a leitura (aqui, 'facilitar a leitura' pode ser entendido como critério). Quando analisamos a concepção do design de um prédio, também vemos princípios que garantem consistência: Mies van der Rohe, considerado um dos mestres do Movimento Moderno e um dos precursores do Estilo Internacional, teria rompido com séculos de arquitetura tradicional com o famoso Pavilhão Alemão (1929), mas dado início a estilos e movimentos que, por sua vez, possuem sua consistência interna ao recorrer a estilos geométricos e minimalistas. Já quando pensamos em sistemas de visualização (e, também, narrativas online contemporâneas), o raciocínio da consistência é difícil de ser aplicado. 'Os diferentes glifos, codificações, tamanhos, estilos, cores, tipografia, layout, espaço em branco e interações reduzem a capacidade de tomar o que é aprendido em uma parte da aplicação e usar em outra' (Brath e Banissi, 2016, tradução nossa, p.3). Há, ainda, influência de outras variáveis, como o objetivo de comunicação, o meio de transmissão e o público, que são dificilmente replicáveis. Dessa forma, é compreensível que os conceitos que fundamentam o design rationale não sejam expostos com frequência por seus elaboradores - mesmo em pesquisas de desenvolvimento de visualizações. Todavia, entendemos que há de se construir um caminho alternativo, uma vez que a documentação do design rationale pode ser útil, conforme entende Brath e Banissi (2016), para avaliar se as suposições de design aplicadas estão aderentes aos conhecimentos técnicos da área.

Destacamos, ainda, a necessidade de aprofundar estudos sobre a aplicação de técnicas e ferramentas de acordo com o tipo de experiência desejada. Observe a figura 1.1. Na primeira questão, sobre qual tamanho deveria ser a barra de rolagem do site em questão, a opção pequena corresponde ao critério tela compacta. Ou seja: caso o objetivo seja planejar a navegação do site em telas pequenas, como celulares ou, ainda, caso trate-se de um design de página minimalista, aconselhável seria optar por barra de rolagem pequena. Caso a preferência seja por permitir maior facilidade para clicar na barra de rolagem com o mouse - 0 que pode ser um fator que incrementa a usabilidade a depender do público -, a opção mais indicada para o tamanho seria grande. Agora vamos buscar resposta tão objetiva quanto essa para uma questão que gravita mais frequentemente entre os desenvolvedores dos conteúdos que aqui estudamos: o debate rolagem ou clique. Parece tendência desses conteúdos fazerem conteúdos que são longos e o leitor vai navegando por meio da rolagem: trata-se da verticalização identificada por Canavilhas, Baccin e Satuf (2017). Mas não há evidências da superioridade técnica dessa preferência. McKenna et al. (2017), por exemplo, alegam que enquanto a equipe do New York Times defende que as histórias de rolagem teriam maior consumo que aquelas que demandam que o leitor fique clicando para continuar a leitura, eles não puderam comprovar essa diferença por meio das pesquisas que conduziram.

Por fim, destacamos que, embora haja uma aparente tendência de utilizar diversas técnicas e ferramentas para construir histórias de dados, não há evidência de que a utilização combinada desses diferentes recursos é apreciada pelo usuário.

\section{Referências}

Appelgren, E., \& Nygren, G. (2014). Data Journalism in Sweden: Introducing new methods and genres of journalism into "old" organizations. Digital Journalism, 2(3), pp. 394-405.

Bakker, P. (2014). Mr. Gates Returns: Curation, community management and other new roles for journalists. Journalism Studies, 15(5), pp. 596-606.

Anais do 9 Congresso Internacional de Design da Informação । CIDI 2019

Proceedings of the 9th Information Design International Conference

Anais do $9^{\circ}$ Congresso Nacional de Iniciação Científica em Design da Informação | CONGIC 2019

Proceedings of the $9^{\text {th }}$ Information Design Student Conference 
Berret, C., Phillips, C., \& Coll, S. (2016). Teaching data and computational journalism.

Bostock, M., Ogievetsky, V., \& Heer, J. (2011). D3 Data-Driven Documents. IEEE Transactions on Visualization and Computer Graphics, 17, 2301-2309. https://doi.org/10.1109/tvcg.2011.185

Brath, R., \& Banissi, E. (2016). Evaluation of Visualization by Critiques. Proceedings of the Beyond Time and Errors on Novel Evaluation Methods for Visualization - BELIV '16, pp. 19-26.

Canavilhas, J., Baccin, A., \& Satuf, I. (2017). Era pós-PC: A nova tessitura da narrativa jornalística na web.

Coddington, M. (2015). Clarifying Journalism's Quantitative Turn: A typology for evaluating data journalism, computational journalism, and computer-assisted reporting. Digital Journalism, 3(3), pp. 331-348.

Conlen, M., \& Heer, J. (2018). Idyll: A Markup Language for Authoring and Publishing Interactive Articles on the Web. (UIST '18), 13.

Davenport, L. D., Fico, F., \& DeFleur, M. H. (2002). Computer-Assisted Reporting in Classrooms: A Decade of Diffusion and a Comparison to Newsrooms. Journalism and Mass Communication Educator, 57(1), pp. 6-22.

Diakopoulos, Nicholas. (2011, abril 22). A Functional Roadmap for Innovation in Computational Journalism.

Dykes, B. (2016, março 31). Data Storytelling: The Essential Data Science Skill Everyone Needs.

Fink, K., \& Anderson, C. W. (2015). Data Journalism in the United States: Beyond the "usual suspects". Journalism Studies, 16(4), pp. 467-481.

Gray, J., Chambers, L., \& Bounegru, L. (2012). The Data Journalism Handbook: How Journalists Can Use Data to Improve the News (1 ed).

Groot Kormelink, T., \& Costera Meijer, I. (2015). Truthful or Engaging?: Surpassing the dilemma of reality versus storytelling in journalism. Digital Journalism, 3(2), pp. 158-174.

Gynnild, A. (2014). Journalism innovation leads to innovation journalism: The impact of computational exploration on changing mindsets. Journalism: Theory, Practice \& Criticism, 15(6), pp. 713-730.

Hewett, J. (2016). Learning to teach data journalism: Innovation, influence and constraints. Journalism: Theory, Practice \& Criticism, 17(1), pp. 119-137.

Howard, A. B. (2014). The Art and Science of Data-Driven Journalism. Academic Commons.

Jacobson, S., Marino, J., \& Gutsche, R. E. (2016). The digital animation of literary journalism. Journalism: Theory, Practice \& Criticism, 17(4), pp. 527-546.

Kerrane, K., \& Yagoda, B. (1998). The Art of Fact: A Historical Anthology of Literary Journalism. Simon and Schuster.

Knight, M. (2015). Data journalism in the UK: A preliminary analysis of form and content. Journal of Media Practice, 16(1), pp. 55-72.

Lee, B., Riche, N. H., Isenberg, P., \& Carpendale, S. (2015). More Than Telling a Story: Transforming Data into Visually Shared Stories. IEEE Computer Graphics and Applications, 35, pp. 84-90.

MacLean, A., M. Young, R., Bellotti, V., \& P. Moran, T. (1991). Questions, Options, and Criteria: Elements of Design Space Analysis. Human-Computer Interaction, 6, pp. 201-250.

Mancini, L., \& Vasconcellos, F. (2016). Jornalismo de Dados: Conceito e categorias. Fronteiras - estudos midiáticos, 18(1). 
McKenna, S., Henry Riche, N., Lee, B., Boy, J., \& Meyer, M. (2017). Visual Narrative Flow: Exploring Factors Shaping Data Visualization Story Reading Experiences. Computer Graphics Forum, 36(3), pp. 377-387.

Meyer, P. (1973). Precision Journalism: A Reporter's Introduction to Social Science Methods.

Moritz, D., Wang, C., Nelson, G. L., Lin, H., Smith, A. M., Howe, B., \& Heer, J. (2018). Formalizing Visualization Design Knowledge as Constraints: Actionable and Extensible Models in Draco. IEEE Transactions on Visualization and Computer Graphics, 1-1.

Müller, O., Junglas, I., Brocke, J. vom, \& Debortoli, S. (2016). Utilizing big data analytics for information systems research: Challenges, promises and guidelines. European Journal of Information Systems, 25(4), pp. 289-302.

Nossek, H., \& Berkowitz, D. (2006). Telling "our" story through news terrorism: mythical newswork as journalistic practice in crisis. Journalism Studies, 7(5), pp. 691-707.

Ojo, A., \& Heravi, B. (2018). Patterns in Award Winning Data Storytelling: Story Types, Enabling Tools and Competences. Digital Journalism, 6(6), pp. 693-718.

Parasie, S., \& Dagiral, E. (2013). Data-driven journalism and the public good: "Computerassisted-reporters" and "programmer-journalists" in Chicago. New Media \& Society, 15(6), pp. 853-871.

Segel, E., \& Heer, J. (2010). Narrative Visualization: Telling Stories with Data. IEEE Transactions on Visualization and Computer Graphics, 16(6), pp. 1139-1148.

Shim, H. (2014). Narative journalism in the contemporary newsroom: The rise of new paradigm in news format? Narrative Inquiry, 24(1), pp. 77-95.

Stavelin, E. (2014). Computational Journalism. When journalism meets programming (The University of Bergen).

Träsel, M. (2014). Jornalismo guiado por dados: Aproximações entre a identidade jornalística e a cultura hacker. Estudos em Jornalismo e Mídia, 11(1), 291.

Westerlund, B. (2009). Design Space Exploration. Kalmar University.

Williams, W. S. (1997). Computer-Assisted Reporting and the Journalism Curriculum. Journalism \& Mass Communication Educator, 52(1), pp. 67-71.

Wing, J. (2006). Computational thinking. Commun. ACM, 49, pp. 33-35.

Wolfe, T. (1973). The New Journalism (Underlining/margin Notes edition). New York: Harper and Row.

Young, M. L., Hermida, A., \& Fulda, J. (2018). What Makes for Great Data Journalism?: A content analysis of data journalism awards finalists 2012-2015. Journalism Practice, $12(1)$, pp. 115-135.

\section{Sobre as autoras}

Tajla Medeiros, Mestra, UnB, Brasil <tajla.medeiros@gmail.com>

Virgínia Tiradentes Souto, PhD, UnB, Brasil<v.tiradentes@gmail.com> 